\title{
Recognition of Resveratrol by the Human Estrogen Receptor-Alpha: A Molecular Modeling Approach to Understand Its Biological Actions
}

\author{
Abdalla M. El-Mowafy ${ }^{a}$ Laila A. Abou-Zeidc Ivan Edafioghob \\ Departments of a Applied Therapeutics and bPharmacy Practice, Faculty of Pharmacy, Kuwait University, Kuwait; \\ 'Department of Medicinal Chemistry, Faculty of Pharmacy, Mansoura University, Mansoura, Egypt
}

\section{Key Words}

Human estrogen receptor- $\alpha \cdot$ Resveratrol .

Phytoestrogens $\cdot$ Molecular modeling

\begin{abstract}
Objectives: Resveratrol (RSVL) is an edible phytoestrogen with multifaceted health benefits that may originate from binding to the estrogen receptors. Despite its structural similarity to the estrogen receptor- $\alpha(E R \alpha)$ agonist diethylstilbestrol (DES), RSVL showed distinct biological profiles in estrogen-responsive biological systems. The molecular basis of such biological profiles has been undefined. Methods: We considered possible orientations for RSVL in ER $\alpha$ binding pocket. These conformations have been analyzed based on: (i) alignment with the key pharmacophoric elements of DES; (ii) computational energy of interaction, and (iii) pattern of accommodation at the ER $\alpha$ binding pocket. The characteristics of the most favored RSVL orientation have been compared with those of DES. Results: Both RSVL and DES interacted with the catalytic amino acid triad of the ER $\alpha$ pocket (His524, Arg394 and Glu 353). However, unlike the Era agonists DES and estradiol $\left(E_{2}\right)$, RSVL formed three additional hydrogen bonds with Gly521 and Leu525, two paramount ligand recognition residues, and with Met343 at the ER $\alpha$ binding cavity. Lastly, RSVL displayed a more favorable energy of interaction with the ER $\alpha$ binding cav-
\end{abstract}

\section{KARGER}

Fax +41613061234

E-Mail karger@karger.ch

www.karger.com

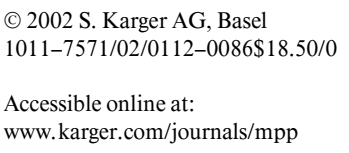

ity. Conclusions: The present study suggests, for the first time, that RSVL is well recognized by the human ER $\alpha$ but in a manner distinct from the pure agonists DES and $E_{2}$. These variations may well entail the unique biological responses of RSVL in ER-responsive systems.

Copyright $\odot 2002$ S. Karger AG, Basel

\section{Introduction}

Resveratrol (trans-3,4',5-trihydroxystilbene, RSVL; fig. 1) is a naturally occurring polyphenolic stilbene that is commonly found in grapes and berries [1]. Its availability in red, but not white wine suggests that RSVL protects against cardiovascular diseases in certain populations, such as the French and Greek, despite having a diet that is rich in fat [2]. This dogma, commonly designated as 'the French paradox of red wine', has triggered an explosion of research in the last decade, which revealed that RSVL confers antimitotic/antiatherotic, antineoplastic, antioxidant, antiplatelet, and anti-inflammatory actions [3-7]. A great deal of these biological profiles was attributed to interaction with the estrogen receptor machinery [8]. Not surprisingly, therefore, RSVL was classified as a phytoestrogen. The phytoestrogen family is a group of nonsteroidal dietary products which can modulate the function of estrogen-dependent biological systems [9]. Although the biological actions of phytoestrogens are well established in
A.M. El-Mowafy, PhD

Department of Applied Therapeutics, Faculty of Pharmacy

Kuwait University, PO Box 24923

13110 Safat (Kuwait)

Tel. +965 534 7926, Fax +965 534 7923, E-Mail aelmowafy@hsc.kuniv.edu.kw 


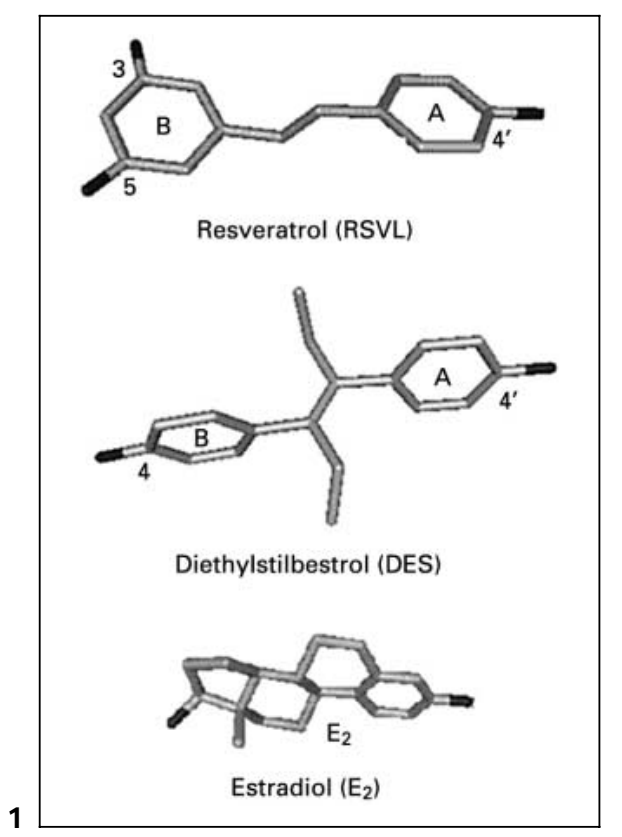

Fig. 1. Energy-minimized structure of RSVL and the crystallographic structure of DES and $\mathrm{E}_{2}$.

Fig. 2. a Flat-ribbon presentation of the crystallographic structure of DES-ER $\alpha$-LBD showing DES (space-filling) and the catalytic triad residues (His524, Arg394 and Glu353, dark structures) at the ER binding domain. $\mathbf{b}$ Crystal structure of DES at the ER $\alpha$ binding pocket showing $\mathrm{H}$-bonding interactions of DES with the catalytic triad residues (dashed lines). Numbers indicate the length of HB in ångström.

humans and animals, their exact mechanism of action remains unclear. Intriguingly, RSVL bears the closest structural features to the estrogen receptor- $\alpha(E R \alpha)$ agonist diethylstilbestrol (DES, fig. 1). However, it displayed a mixed agonist/antagonist activity on the ER $\alpha[10]$. The underpinnings of these responses are barely understood. Currently, a molecular modeling study was conducted to help clarify these ambiguities. The molecular modeling profiles of RSVL have been delineated and further compared with those of DES and the prototype estradiol $\left(\mathrm{E}_{2}\right.$, fig. 1). This study is the first to highlight the mode of recognition of RSVL by the human ER $\alpha$.

\section{Computational Methods}

Receptor Structure

Starting coordinates of the human ER $\alpha$ ligand-binding domain in complex with DES (DES-ER $\alpha$-LBD, code ID 3ERD) was obtained from the Protein Data Bank of Brookhaven National Laboratory (fig. 2) [11].

Recognition of Resveratrol by the Human

Estrogen Receptor-Alpha

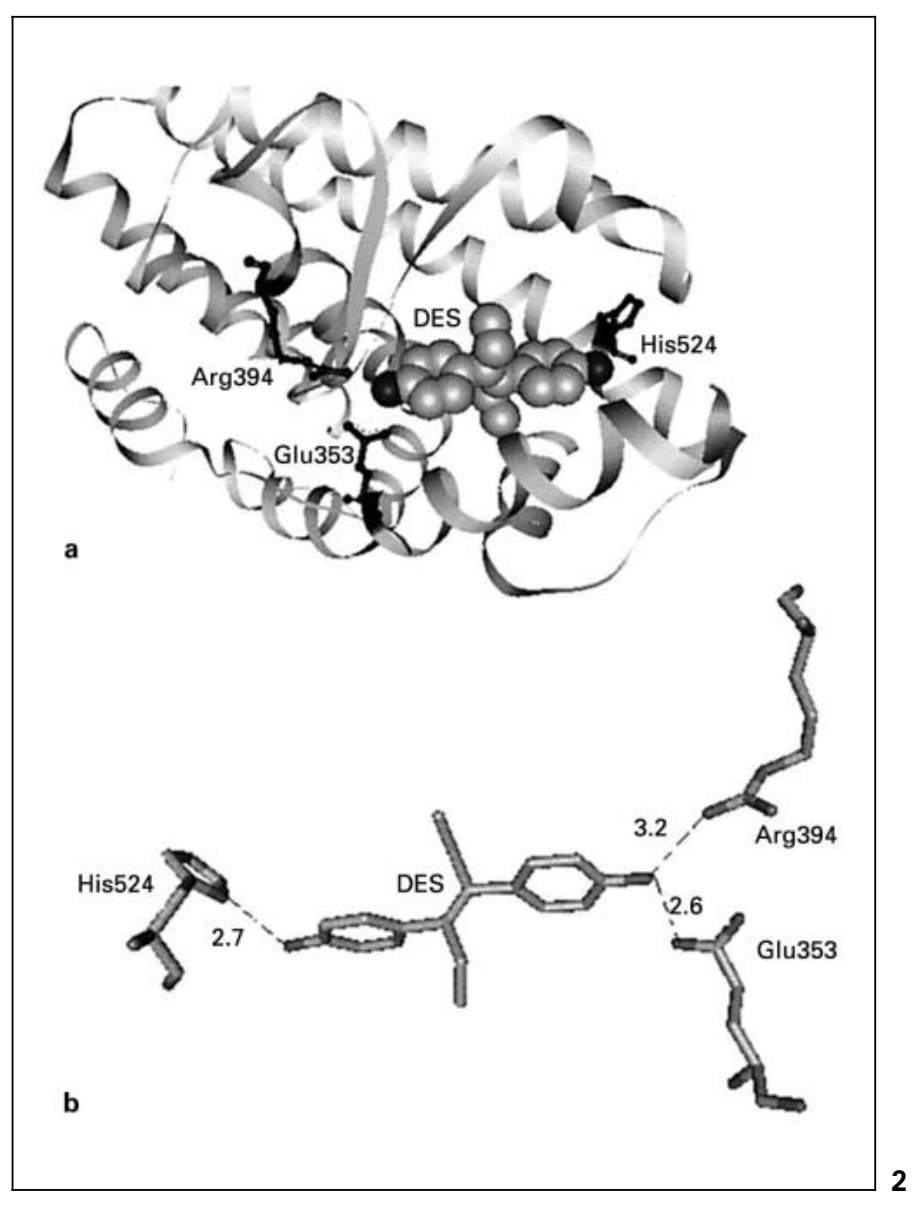

Molecular Structure of RSVL

The starting conformation for trans-RSVL used for receptor docking studies was generated from random conformational search performed using the AMBER force field as implemented in GROMOS [12]. The resulting lowest-energy conformer was then used for docking studies. Charge calculations were determined using the semiempirical quantum mechanics method 'AMI' as implemented in HyperChem 6 [13]. The resulting lowest-energy conformer of RSVL was prepositioned on DES at the ER $\alpha$ binding domain (fig. 2) using a least-squares multifitting of the selected atoms within the DES ligand (pharmacophoric elements: A, B rings and the stilbene bond) [14]. Once prepositioned, DES was deleted and RSVL was optimally docked in the ER $\alpha$ binding pocket in the possible orientations (as detailed in the text) using the Flexidock routine implemented in PowerFit [15] (fig. 3). Both hydrogen bond donors and acceptors within the pocket surrounding the ligand (Glu353, Arg394 and His524), the ligand itself and the torsional bonds were defined. The best docked receptor-ligand complex from Flexidock then underwent a three-step minimization: first, the nonring torsional bonds of the ligand were minimized in the context of the receptor, followed by minimization of the side chain residues within $8 \AA$ of the ligand while holding the backbone and residues Glu353 and Arg394 fixed. Finally, energy minimization followed by geometric optimization of both the ligand and receptor was conducted until an energy convergence of 
Fig. 3. All possible orientations of the lowest energy-minimized RSVL. The structures were generated by rotation around the stilbenic double bond and/or exchange of the phenolic rings.

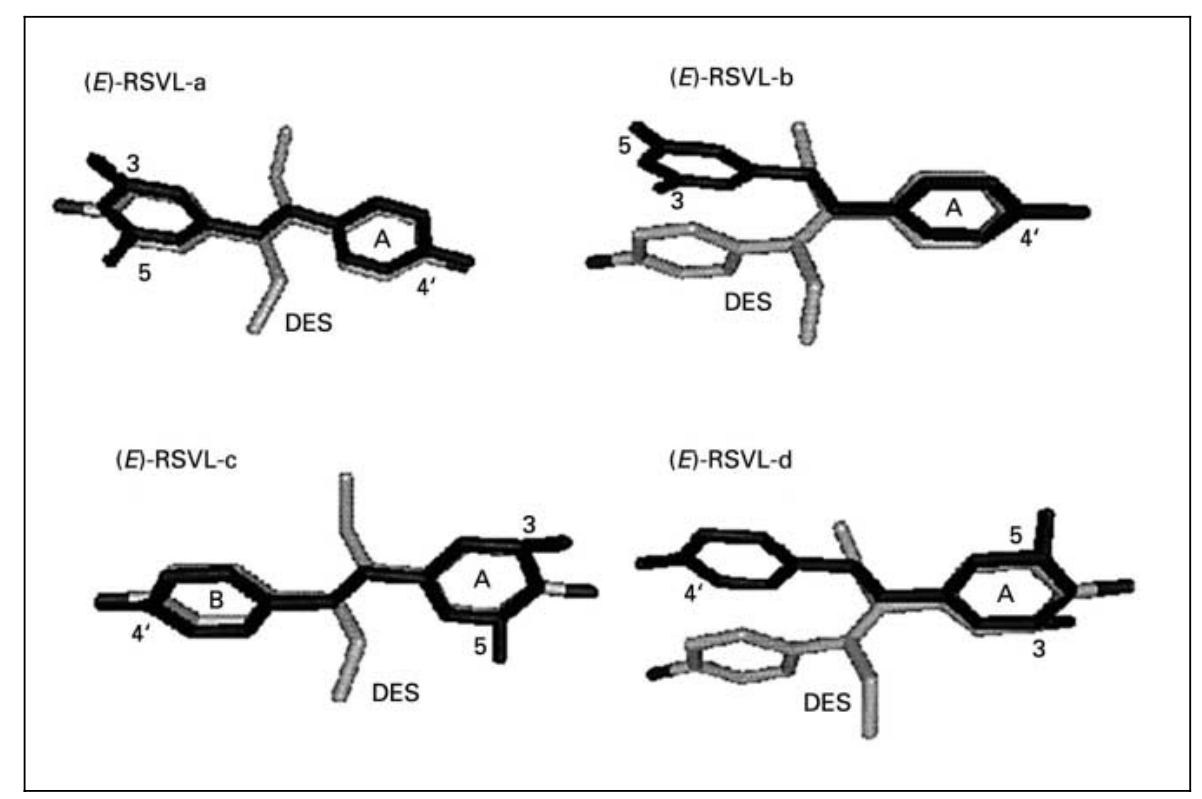

$0.05 \mathrm{kcal} / \mathrm{mol} \AA$ was reached $[16,17]$. Energy of interaction was calculated for each RSVL-ER $\alpha$ model after minimization as the difference between the energy of the complex and individual energies of the receptor and the ligand (RSVL) $[18,19]$ :

$$
\mathrm{E}_{\text {interaction }}=\mathrm{E}_{\text {Complex }}-\left(\mathrm{E}_{\text {Ligand }}+\mathrm{E}_{\text {Receptor }}\right) \text {. }
$$

\section{Results and Discussion}

RSVL is an edible stilbene of plant origin with a myriad of beneficial biological effects [1-3]. An elegant population study demonstrated that dietary intake of RSVL can attain plasma levels that are evidently biologically active [20]. Owing to striking structural similarity to the ER agonist DES, RSVL has been established as a phytoestrogen [2]. Subsequent studies for RSVL revealed distinct ligand characteristics that combined both agonistic and/or antagonistic effects on the estrogen machinery. In a detailed study that employed extensive biological testing protocols, two structurally unrelated phytoestrogens, RSVL and naringen (a flavanoid), were comparatively investigated [21]. Both ligands exhibited agonistic activity in the absence of $E_{2}$. However, only RSVL displayed antagonistic activity when present with $\mathrm{E}_{2}$. Thus, based on analysis of the structure-activity relationship of these phytoestrogens and other ER ligands, it was concluded that differences in ligand behavior are primarily related to the 'ligand structure' [21]. Moreover, recognition and interpretation of ligands at the ligand-binding pocket of
$\mathrm{ER} \alpha$ have also been ascribed to interplay between the shape of the ligand and the amino acid (aa) residues that support ligand accommodation at the receptor binding cavity.

Given the structural features of ER $\alpha$ agonists, such as DES and $E_{2}$, a hydroxyl group in the phenolic A ring appears essential for agonist recognition and activity [22]. Not surprisingly, biological studies with RSVL have also indicated that its 4'-hydroxyl group is absolutely required for its pharmacological actions such as inhibition of cell proliferation and scavenging of free radicals [22-24]. Considering the molecular modeling determinants of DES and RSVL, it appears the they share two phenolic rings (A and $\mathrm{B}$ ) and a stilbenic bond. However, the $\mathrm{B}$ ring for DES carries only one 4-hydroxyl group versus two (3,5)-dihydroxyl groups for RSVL (fig. 3). Therefore, other orientations for RSVL at the binding domain have to be evaluated, especially because a crystal structure for RSVL-ER $\alpha$ complex is lacking. In total, four possible orientations for RSVL can be generated by rotation about the stilbenic bond and/or exchanging location of monophenolic and diphenolic rings (fig. 3). We employed the DES-phenolic ring A as a guiding tool to correctly position these RSVL models at the ER $\alpha$ binding domain $[13,16]$. The energy-minimized models were comparatively studied based on their features of accommodation at the ER $\alpha$ binding pocket.

Table 1 demonstrates that the RSVL-a mode had superior hydrogen bonding profiles and showed the lowest 
Fig. 4. a Flat-ribbon presentation of $E R \alpha$ in complex with RSVL-a (space-filling). The defined residues (dark structures) are those involved in $\mathrm{H}$ bonding with RSVL-a. b Binding mode of (E)-RSVL-a docked and minimized in the ER $\alpha$ binding pocket showing residues involved in its recognition. Dashed lines indicate $\mathrm{H}$-bond formation and numbers indicate their length in ångström.

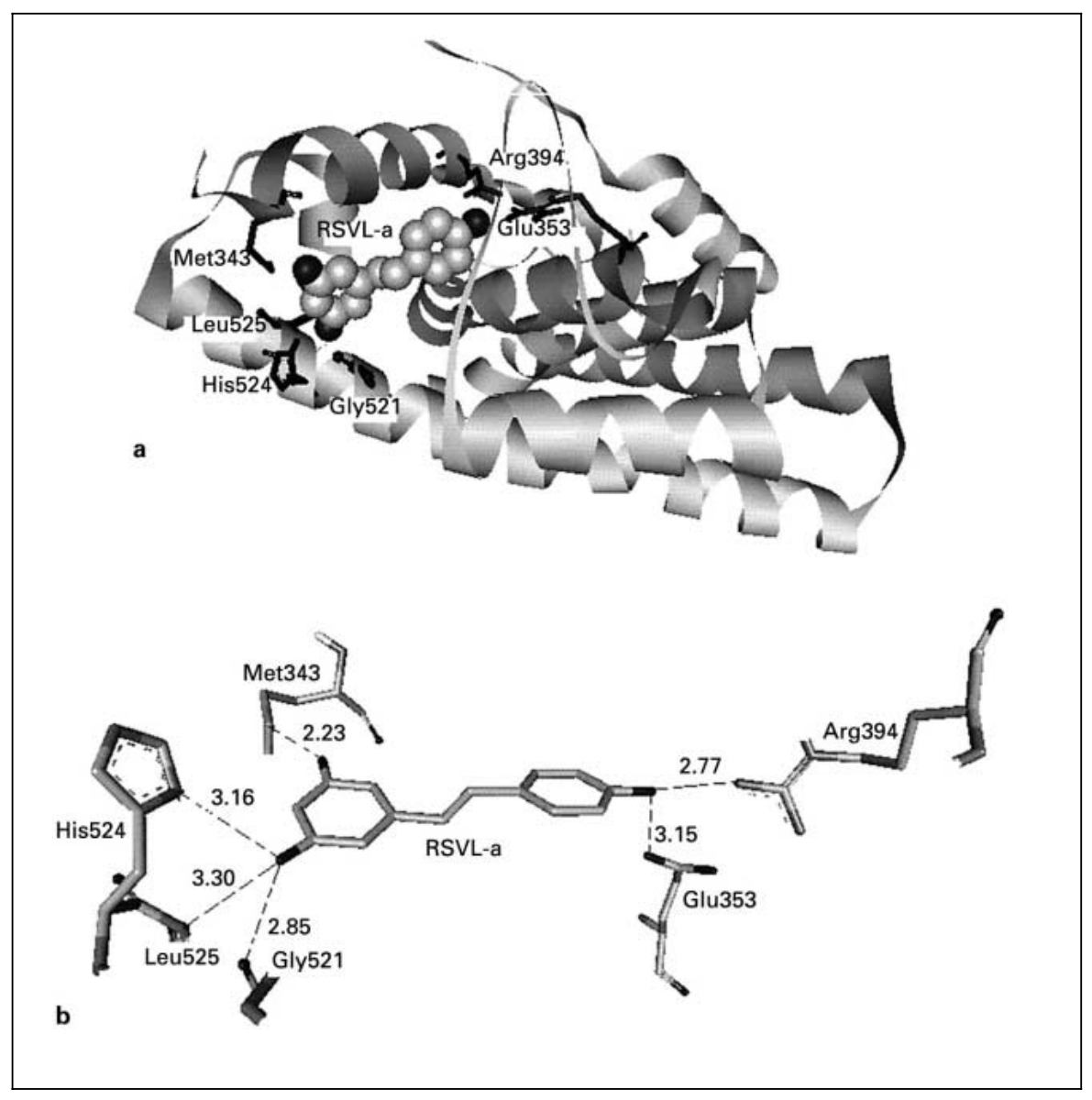

energy of interaction with the receptor (fig. 4). Model 'RSVL-b', resulting from rotation around the stilbenic bond, had no capacity to form $\mathrm{H}$ bonds with His524 or Leu525 (fig. 5). The two latter residues, as established by mutation studies, are pivotal for ligand interaction and recognition [25]. Also, the energy of interaction of RSVL-b with the receptor $(-8.3$ vs. $-16.3 \mathrm{kcal} / \mathrm{mol}$ for RSVL-a) suggests less favorable accommodation within the receptor binding pocket. The other two orientations, RSVL-c and RSVL-d, resulting from switching the position of rings $\mathrm{A}$ and $\mathrm{B}$, lacked the ability to interact with Arg394, a crucial ligand-binding residue [26]. Besides, as reflected by their energy of interaction, RSVL-c and RSVL-d were poorly accommodated within the receptor binding pocket (table 1). Apparently, exchanging rings A and $\mathrm{B}$ increased the steric repulsion and adversely affected their complementarity with the receptor. These studies suggest that RSVL-a mode led to the most favorable interaction with the ER $\alpha$. Likewise, alignment studies with DES-ER crystal structure indicated that RSVL-a
Table 1. Calculated ligand binding interaction energy at the ER $\alpha$ binding cavity $(\mathrm{kcal} / \mathrm{mol})$

\begin{tabular}{lccc}
\hline Ligand & $E_{\text {ligand }}$ & $E_{\text {complex }}$ & $E_{\text {interaction }}$ \\
\hline RSVL-a & -3.7 & -298.0 & -16.3 \\
RSVL-b & -3.7 & -290.0 & -8.3 \\
RSVL-c & -3.7 & -285.0 & -3.3 \\
RSVL-d & -3.7 & -283.0 & -1.3 \\
DES & -10.5 & -298.6 & -10.1 \\
\hline
\end{tabular}

Energy of ligand free receptor $=-278.0 \mathrm{kcal} / \mathrm{mol} \mathrm{E}_{\text {interaction }}=$ $\mathrm{E}_{\text {complex }}-\left(\mathrm{E}_{\text {ligand }}+\mathrm{E}_{\text {receptor }}\right)$

orientation is far superior to the rest of the RSVL orientations. Accordingly, the modeling characteristics of RSVLa and DES have been comparatively evaluated. For both ligands, the 4'-hydroxyl group contributes a hydrogen bond with the receptor's glutamate (Glu353). Moreover, 


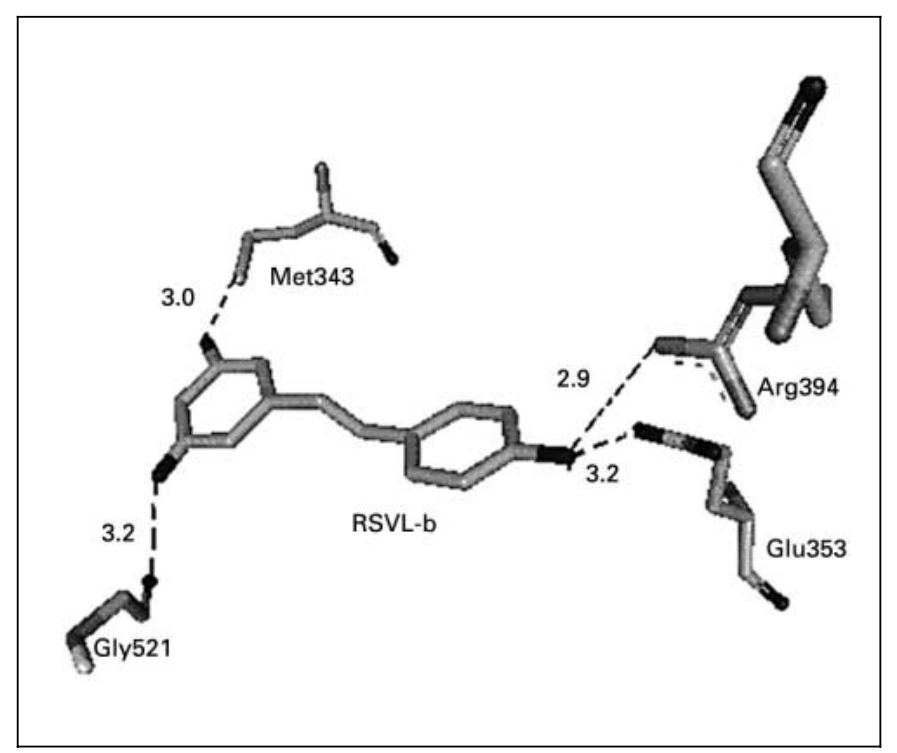

Fig. 5. Binding mode for RSVL-b docked and minimized in the ER $\alpha$ binding pocket, showing residues involved in its recognition. Dashed lines indicate H-bond formation and numbers indicate their length in ångström.

this same hydroxyl group is additionally recognized by the side chain of the conserved arginine (Arg394), which correctly orients and braces the ligand at the binding pocket of the receptor. Histidine (His524) is a third important catalytic residue which interacts, via its delta nitrogen, with the 4-hydroxyl group of the B ring of DES or the 5hydroxyl group of RSVL (fig. 2 and 4, respectively). This binding mode of RSVL and DES with the catalytic triad aa is similar to that observed for the prototype $\mathrm{E}_{2}$ (fig. 6) [26]. Thus, it appears that all hydroxyl groups from all ligands were actively engaged in $\mathrm{H}$ bonding (fig. 2, 4, 6). Uniquely, however, RSVL-a developed three additional $\mathrm{H}$ bonds with Gly521, Leu525 and Met343. Evidently, such additional recognition for RSVL-a results from the altered orientation of its B ring hydroxyl groups $(3,5-\mathrm{OH})$, as compared to that of DES (4-OH, fig. 7). As established by mutation and receptor binding studies with $\mathrm{E}_{2}$, Gly521 and Leu525 have been identified as key components for interaction and/or interpretation of ligands at the ER $\alpha$ binding cavity [25]. These residues were hydrogenbonded to the 5-OH group of RSVL's B ring. The pocket residue, Met343, has been identified as a merely hydrophobic van der Waal contact point for $\mathrm{E}_{2}$ [26]. Currently, this residue was recognized by RSVL through $\mathrm{H}$ bond interaction, due to the presence of $3-\mathrm{OH}$ group in its $\mathrm{B}$ ring. This latter interaction further constitutes a unique recognition mode for RSVL that was not observed for other ligands modeled at the binding domain of the ER $\alpha$ receptor [27]. However, the impact and implications of this additional interaction remain to be substantiated via experimental receptor mutation studies. Lastly, RSVL exhibited a more favorable energy of binding than DES $(-16.3 \mathrm{vs} .-10.1 \mathrm{kcal} / \mathrm{mol})$. This is likely conferred by the differential ligand interaction with the pocket aa residues. Previous molecular modeling studies with ER ligands suggested that more favorable interaction energy associates with partially antagonistic rather than purely agonistic activity, which conforms to the present observations as well as the behavior of RSVL in ER $\alpha$-responsive systems $[10,18]$.

Although the present study is apparently a merely computer-based evaluation, its biological relevance is warranted. That is, it relied on a documented three-dimensional topography of the receptor and actual structure/ geometry of its aa pocket residues. The present notion that RSVL mimicked the binding of DES to the catalytic triad aa (His524, Arg394 and Glu353) conforms well to its actions as an agonist in estrogen-free systems. On the other hand, the additional recognition of RSVL by three other ER $\alpha$ pocket residues (Gly521, Leu525 and Met343) may influence the stability of the formed receptor-ligand complex, and the readiness of this complex to reach the nucleus or to interact with the $\mathrm{E}_{2}$ nuclear machinery. This view is reinforced by experimental findings on activation of ERra in human HepG2 liver and U2 osteogenic cancer cells. Thus, RSVL was an agonist that stimulated ER $\alpha$ dependent luciferase activity in U2 cells transfected with ER activation factor-1 (ER-AF1), but not with ER-AF2. Another phytoestrogen, naringen, activated only HepG2 cells transfected with ER-AF1. In HepG2 cells, only RSVL attenuated the $\mathrm{E}_{2}$-evoked, ER $\alpha$-mediated luciferase reporter activity [21]. Accordingly, it is tempting to hypothesize that coexistence of two ligands that variably bind the pocket aa residues could adversely affect the binding and responsiveness to the purely agonistic ligand, $E_{2}$. These perspectives may well agree with the observed agonist/antagonist behavior of RSVL in some biological systems [28]. Indeed, site-directed mutations of aa residues of the ER $\alpha$ pocket clearly demonstrated that antagonists like hydroxytamoxifen display preference to bind some pocket residues other than those best recognizing the full agonist $\mathrm{E}_{2}$. However, some aa residues were inevitably required for actions of both agonists and antagonists [25], as similarly demonstrated in the present work. In this context, it is worthwhile mentioning that RSVL is not merely an ER ligand, as it also elicits a myriad of actions 
Fig. 6. a Flat-ribbon presentation of the crystallographic structure of $\mathrm{E}_{2}$-Er $\alpha$-LBD showing $E_{2}$ (space-filling) and the catalytic triad residues (His524, Arg394 and Glu353, dark-stick) at the ER binding domain. b Crystal structure of $E_{2}$ at the ER $\alpha$ binding pocket showing $\mathrm{H}$-bonding interactions with the catalytic triad residues (dashed lines). Numbers indicate the length of HB in ångström.

Fig. 7. Superposition of the X-ray structure of DES and the energy-minimized RSVL at the active site of ER $\alpha$. The overlay demonstrates that DES and RSVL have common binding to the catalytic aa triad. Besides, RSVL exhibited inimitable interaction with other key residues (Gly521, Leu525 and Met343). Dashed lines represent HB interaction of RSVL, and solid lines represent those of DES.

Recognition of Resveratrol by the Human Estrogen Receptor-Alpha
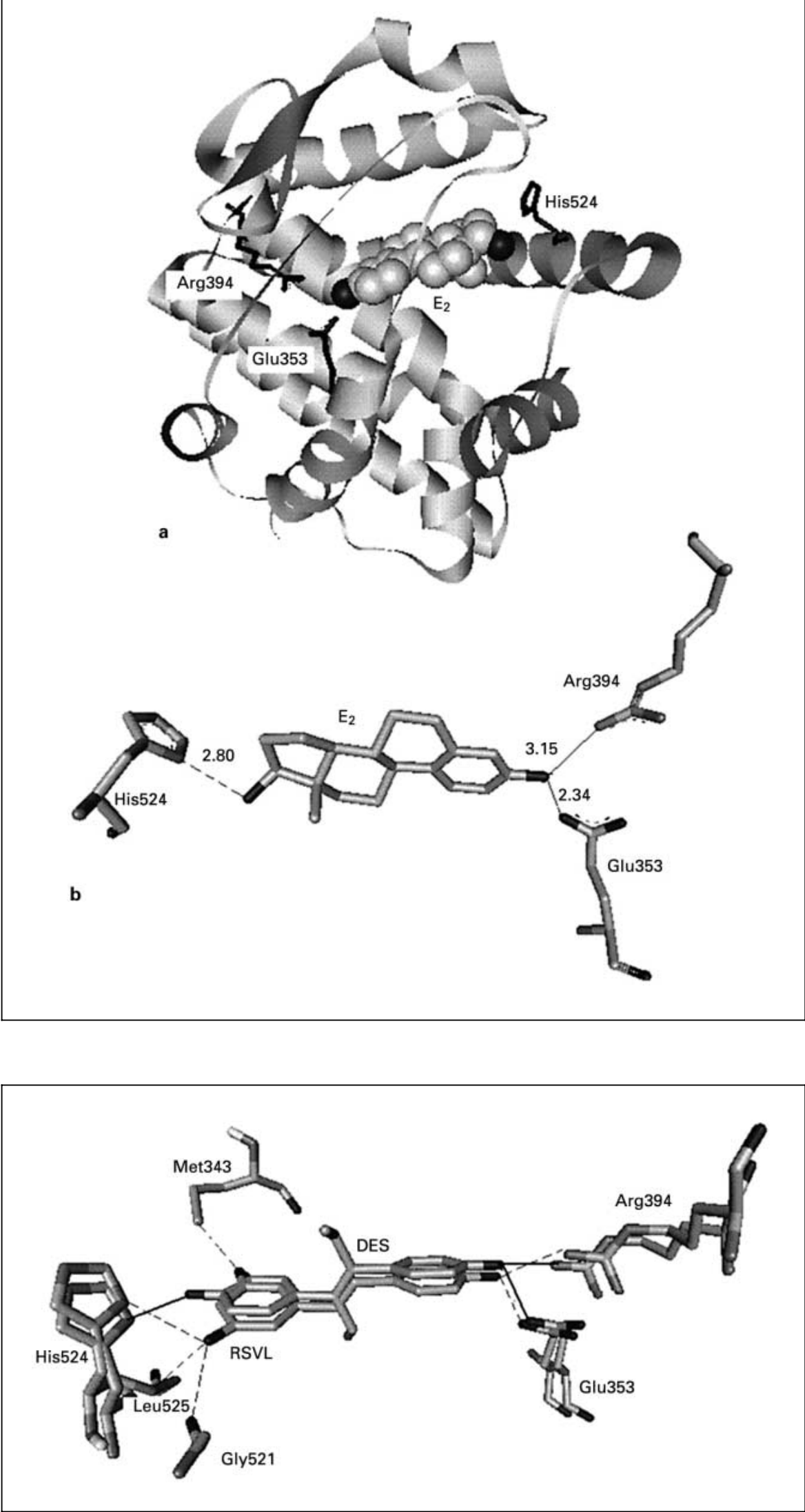

Med Principles Pract 2002;11:86-92 
pertinent to other cellular effectors, including enzymes [1] and cell cycle checkpoints [29]. Thus, RSVL was established as a versatile agent that can act by both ER-dependent and -independent mechanisms, occasionally even in the same biological system [29].

\section{Conclusion}

Altogether, the present study highlights major differences in the modeling characteristics of pure estrogen agonist DES and the biologically active ER agonist/antagonist RSVL; these variations may entail distinct patterns of ligand recognition 'footprinting' that subsequently dictate the ligand's biological actions.

\section{References}

1 El-Mowafy AM, White RE: Resveratrol inhibits MAPK activity and nuclear translocation in coronary artery smooth muscle: Reversal of endothelin-1 stimulatory effects. FEBS Lett 1999;451:63-67.

2 Kopp P: Resveratrol, a phytoestrogen found in red wine: A possible explanation for the conundrum of the 'French paradox'? Eur J Endocrinol 1998;138:619-620.

3 Fremont L: Biological effects of resveratrol. Life Sci 2000;66:663-673.

4 Lu R, Serrero G: Resveratrol, a natural product derived from grape, exhibits antiestrogenic activity and inhibits the growth of human breast cancer cells. J Cell Physiol 1999;179:297-304.

5 Basly JP, Marre-Fournier F, LeBail JC, Habrioux G, Chulia AJ: Estrogenic/antiestrogenic and scavening properties of (E)- and (Z)-resveratrol. Life Sci 2000;66:769-777.

6 Gentilli M, Mazoit JX, Bouaziz H, Fletcher D, Casper RF, Benhamou D, Savouret JF: Resveratrol decreases hyperalgesia induced by carrageenan in the rat hind paw. Life Sci 2001;68 1317-1321.

7 Jang M, Cai L, Udeani GO, Slowing KV, Thomas $\mathrm{CF}$, Beecher CW, Fong HH, Farnsworth NR, Kinghorn AD, Mehta RG, Moon RC, Pezzuto JM: Cancer chemopreventive activity of resveratrol, a natural product derived from grapes. Science 1997;275:218-220.

8 Gehm BD, McAndrews JM, Chien PY, Jameson JL: Resveratrol, a polyphenolic compound found in grapes and wine, is an agonist for the estrogen receptor. Proc Natl Acad Sci USA 1997;94:14138-14143.

9 Stahl S, Chun TY, Gray WG: Phytoestrogens act as estrogen agonists in an estrogen-responsive pituitary cell line. Toxicol Appl Pharmacol 1998;152:41-48.

10 Bowers JL, Tyulmenkov VV, Jernigan SC, Klinge CM: Resveratrol acts as a mixed agonist/antagonist for estrogen receptors alpha and beta. Endocrinology 2000;141:3657-3667.

11 Shiau AK, Barstad D, Loria PM, Cheng L, Kushner PJ, Agard DA, Greene GL: The structural basis of estrogen receptor/coactivator recognition and the antiagonism of this interaction by tamoxifen. Cell 1998;95:927-937.
12 Gunsteren WF, Hünenberger PH, Mark AE, Smith PE, Tironi IG: Computer simulation of protein motion. Comput Phys Commun 1995; 91:305-319

13 Hyperchem: Moleclar Modeling System. Hypercube, Inc. Release 6, Fla, 1999

14 Stauffer SR, Sun J, Katzenellenbogen BS, Katzenellenbogen JA: Acyclic amides as estrogen receptor ligands: Synthesis, binding, activity and receptor interaction. Bioorg Med Chem 2000;8:1293-1316

15 Powerfit: Microsimulations. MSI, Inc, Release 1, NJ 1996.

16 Stauffer SR, Huang Y, Coletta CJ, Tedesco R, Katzenellenbogen JA: Estrogen pyrazoles: Defining the pyrazole core structure and the orientation of substituents in the ligand binding pocket of the estrogen receptor. Bioorg Med Chem 2001;9:141-150.

17 Stauffer SR, Coletta CJ, Tedesco R, Nishiguchi G, Carlson K, Sun J, Katzenellenbogen BS, Katzenellenbogen JA: Pyrazole ligands: Structure-affinity/activity relationships and estrogen receptor-alpha-selective agonists. J Med Chem 2000;43:4934-4947.

18 Lewis DF, Parker MG, King RJ: Molecular modeling of the human estrogen receptor and ligand interaction based on site-directed mutagenesis and amino acid sequence homology. $\mathbf{J}$ Steroid Biochem Mol Biol 1995;52:55-65.

19 Schmidt CR, Sluka JP, Duke KM, Glasebrook AW: Novel nonsteroidal selective estrogen receptor modulators: Carbon and heteroatom replacement of oxygen in the ethoxypiperidine region of raloxifene. Bioorg Med Chem Lett 1999;9:523-528.

20 Bertelli AA; Giovannini L, Giannessi D, Migliori M, Bernini W, Fregoni M, Bertelli A: Antiplatelet activity of synthetic and natural resveratrol in red wine. Int $\mathrm{J}$ Tissue React 1995; 17:1-3.

21 Yoon K, Pellaroni L, Ramamoorthy K, Gaido K, Safe S: Ligand structure-dependent differences in activation of estrogen receptor alpha in human HepG2 liver and U2 osteogenic cancer cell lines. Mol Cell Endocrinol 2000;162: 211-220.
22 Stivala LA, Savio M, Carafoli F, Perucca P, Bianchi L, Maga G, Forti L, Pagnoni UM, Albini A, Prosperi E, Vannini V: Specific structural determinants are responsible for the antioxidant activity and the cell cycle effects of resveratrol. J Biol Chem 2001;276:2258622594.

23 Stojanovic S, Sprinz H, Brede O: Efficiency and mechanism of the antioxidant action of trans-resveratrol and its analogues in the radical liposome oxidation. Arch Biochem Biophys 2001;391:79-89.

24 Karlsson J, Emgard M, Brundin P, Burkitt MJ : trans-Resveratrol protects embryonic mesencephalic cells from tert-butyl hydroperoxide: Electron paramagnetic resonance spin trapping evidence for a radical scavening mechanism. J Neurochem 2000;75:141-150.

25 Ekena K, Weis KE, Katzenellenbogen JA, Katzenellenbogen BS: Different residues of the human estrogen receptor are involved in the recognition of structurally diverse estrogen and antiestrogens. J Biol Chem 1997;272:5069_ 5075.

26 Tanenbaum DM, Wang Y, William SP, Sigler PB: Crystallographic comparison of the estrogen and progesterone receptor's ligand binding domains. Proc Natl Acad Sci USA 1998;95: 5998-6003.

27 Brzozowski AM, Pike AC, Dauter Z, Hubbard RE, Bonn T, Engstrom O, Ohman L, Greene GL, Gustafsson JA, Carlquist M: Molecular basis of agonism and antagonism in the oestrogen receptor. Nature 1997;389:753-758.

28 Bhat KP, Lantvit D, Christov K, Mehta RG, Moon RC, Pezzuto JM: Estrogenic and antiestrogenic properties of resveratrol in mammary tumor models. Cancer Res 2001;61:74567463.

29 Bhat KP, Pezzuto JM: Resveratrol exhibits cytostatic and antiestrogenic properties with human endometrial adenocarcinoma (Ishikawa) cells. Cancer Res 2001;61:6137-6144. 